\title{
Inhibition of Osteoclastogenesis by Mechanically Loaded Osteocytes: Involvement of MEPE
}

\author{
Rishikesh N. Kulkarni • Astrid D. Bakker • \\ Vincent Everts $\cdot$ Jenneke Klein-Nulend
}

Received: 26 April 2010/Accepted: 29 July 2010/Published online: 20 August 2010

(C) The Author(s) 2010. This article is published with open access at Springerlink.com

\begin{abstract}
In regions of high bone loading, the mechanoresponsive osteocytes inhibit osteoclastic bone resorption by producing signaling molecules. One possible candidate is matrix extracellular phosphoglycoprotein (MEPE) because acidic serine- and aspartate-rich MEPE-associated motif peptides upregulate osteoprotegerin $(O P G)$ gene expression, a negative regulator of osteoclastogenesis. These peptides are cleaved from MEPE when relatively more MEPE than PHEX (phosphate-regulating gene with homology to endopeptidases on the $\mathrm{X}$ chromosome) is present. We investigated whether mechanical loading of osteocytes affects osteocyte-stimulated osteoclastogenesis by involvement of MEPE. MLO-Y4 osteocytes were mechanically loaded by 1-h pulsating fluid flow (PFF; $0.7 \pm 0.3 \mathrm{~Pa}, 5 \mathrm{~Hz}$ ) or kept under static control conditions. Recombinant MEPE $(0.05,0.5$, or $5 \mu \mathrm{g} / \mathrm{ml})$ was added to some static cultures. Mouse bone marrow cells were seeded on top of the osteocytes to determine osteoclastogenesis. Gene expression of MEPE, PHEX, receptor activator of nuclear factor kappa-B ligand (RANKL), and $O P G$ by osteocytes was determined after PFF. Osteocytes supported osteoclast formation under static control conditions. Both PFF and recombinant MEPE inhibited osteocyte-stimulated
\end{abstract}

The authors have stated that they have no conflict of interest.

R. N. Kulkarni · A. D. Bakker · V. Everts - J. Klein-Nulend Department of Oral Cell Biology, Academic Centre for Dentistry Amsterdam, University of Amsterdam and VU University Amsterdam, Research Institute MOVE, Van der Boechorststraat

7, 1081 BT Amsterdam, The Netherlands

J. Klein-Nulend $(\square)$

Department of Oral Cell Biology, ACTA-VU University

Amsterdam, Van der Boechorststraat 7, 1081 BT Amsterdam,

The Netherlands

e-mail: j.kleinnulend@vumc.nl osteoclastogenesis. PFF upregulated MEPE gene expression by 2.5 -fold, but not $P H E X$ expression. PFF decreased the RANKL/OPG ratio at 1 -h PFF treatment. Our data suggest that mechanical loading induces changes in gene expression by osteocytes, which likely contributes to the inhibition of osteoclastogenesis after mechanical loading of bone. Because mechanical loading upregulated gene expression of $M E P E$ but not $P H E X$, possibly resulting in the upregulation of $O P G$ gene expression, we speculate that MEPE is a soluble factor involved in the inhibition of osteoclastogenesis by osteocytes.

Keywords Osteocytes - Osteoclastogenesis - Fluid shear stress $\cdot$ MEPE $\cdot$ OPG $\cdot$ RANKL

Bone is a specialized connective tissue that tolerates mechanical loads derived from weight bearing and from muscle contractions [1]. Repeated loads lead to microdamage, which could cause fractures. Therefore, bone is continuously remodeled by osteoclasts and osteoblasts that act in a coordinated fashion [2]. First osteoclasts resorb existing bone tissue, and subsequently osteoblasts refill the gap with new bone tissue. The actions of the osteoblasts and osteoclasts are coordinated by the osteocytes [3-5]. Osteocytes are buried in the bone matrix and are in contact with neighboring osteocytes via long slender cell processes, located in canaliculi, which are filled with pericellular interstitial fluid. These cell processes make direct cell-cell contact not only to neighboring osteocytes, but also to osteoclasts and osteoclast precursors in bone marrow [1]. The three-dimensional network of interconnected cells is present throughout bone. This osteocyte network with its accompanying lacuno-canalicular porosity is likely the site of mechanosensing in bone [2]. In the absence of 
mechanical loading or disuse conditions, stasis of interstitial fluid occurs, leading to a lack of fluid shear stress [2]. Tatsumi et al. [6] have shown that osteocytes produce proosteoclastogenic signals in the absence of mechanical loading leading to stimulation of bone resorption. Alternatively, mechanically loaded osteocytes may produce factors that inhibit osteoclast recruitment, while under disuse conditions osteocytes decrease the production of osteoclast-inhibiting signals [2, 3].

Osteoclasts are multinucleated cells that arise from hemopoietic cells of the monocyte/macrophage lineage [7]. Osteoclast formation in vivo is thought to be induced by direct cell-cell contact of preosteoblastic/stromal cells with monocyte/macrophage osteoclast precursors [7]. Osteoblast lineage cells secrete key molecules responsible for osteoclast differentiation, i.e., receptor activator of nuclear factor kappa-B ligand (RANKL) and macrophage colonystimulating factor (M-CSF). RANKL stimulates osteoclast precursors to commit to the osteoclastic phenotype by binding to its receptor, receptor activator of nuclear factor kappa B (RANK), on the surface of osteoclast precursors [8]. RANKL exists in two forms, a soluble form and a membrane-bound form thought to be responsible for initiating osteoclast formation. RANKL activity is negatively regulated by osteoprotegerin (OPG), which competes with RANK as a soluble receptor. OPG, like RANKL, is produced by cells of the osteoblast lineage [8]. Osteoclast differentiation is inhibited when OPG is relatively more upregulated than RANKL. Thus, the ratio of RANKL to OPG determines osteoclastogenesis [9].

Osteoclastogenesis from mouse bone marrow cells is inhibited by the addition of conditioned medium from mechanically loaded MLO-Y4 osteocytes [3]. Osteocytes can support osteoclastogenesis when co-cultured with RAW264.7 monocyte osteoclast precursor cells, but mechanical loading of osteocytes decreases their osteoclastogenic potential [5]. Soluble factors released by mechanically loaded osteocytes inhibit osteoclastogenesis induced by stromal cells or osteocytes [5]. Osteocytes release RANKL and OPG molecules that regulate osteoclastogenesis. The RANKL/OPG ratio is decreased in mechanically loaded osteocytes compared to nonloaded controls, resulting in inhibition of osteoclastogenesis [5]. It is largely unknown which signaling molecules that are produced by osteocytes in response to mechanical loading can modulate the RANKL/OPG ratio to inhibit osteoclastogenesis. One likely candidate is matrix extracellular phosphoglycoprotein (MEPE).

MEPE is predominantly expressed by osteocytes in human bone, with significant expression by osteocytes within mineralized bone [10]. MEPE mRNA and protein expression in osteocytes is highly responsive to mechanical loading of bone in vivo in a mouse tooth movement model
[11]. Synthetic 23mer peptide fragment of MEPE (AC-100, region 242-264) containing the RGD (Arg-Gly-Asp) integrin binding sequence and SGDG (Ser-Gly-Asp-Gly) glycosaminoglycan-attachment sequence stimulates new bone formation in vitro and in vivo [12]. MEPE serum levels in healthy adult humans vary between 0.02 and $1.3 \mu \mathrm{g} / \mathrm{ml}$. Serum MEPE levels are high when bone mineral density is high and low in aged individuals when bone mineral density is low, suggesting a physiological role of MEPE in bone homeostasis [13]. MEPE protein is most likely cleaved by a cathepsin B-like protease to a highly phosphorylated C-terminal acidic serine- and aspartate-rich MEPE-associated motif (ASARM) peptide in osteocytes. ASARM peptides are cleaved from MEPE when relatively more MEPE than PHEX (phosphate-regulating gene with homology to endopeptidases on the $\mathrm{X}$ chromosome) is present. ASARM peptides increase the expression of OPG, a negative regulator of osteoclastogenesis, in bone marrow stromal cells [14]. MEPE might therefore be an important signaling molecule involved in the regulation of osteoblast and osteoclast activity during bone remodeling. The aim of this study was to investigate whether mechanical loading of osteocytes affects osteocyte-stimulated osteoclastogenesis by involvement of MEPE.

\section{Materials and Methods}

\section{Cell Culture}

MLO-Y4 osteocytes (provided by Dr. Bonewald, San Antonio, TX) [15] were cultured to near confluence in 75- $\mathrm{cm}^{2}$ culture flasks with $\alpha$ minimal essential medium $(\alpha$-MEM) supplemented with $5 \%$ fetal bovine serum (FBS; Gibco, Grand Island, NY), 5\% calf serum (Gibco), $1.25 \mu \mathrm{g} / \mathrm{ml}$ Fungizone (Gibco), $150 \mu \mathrm{g} / \mathrm{ml}$ penicillin (Sigma, St. Louis, MO), and $125 \mu \mathrm{g} / \mathrm{ml}$ streptomycin (Sigma) at $37^{\circ} \mathrm{C}$ and $5 \% \mathrm{CO}_{2}$ in air.

Mouse bone marrow cells were used for the osteoclast formation assay. Briefly, 8-week-old male mice (C57BL/ $6 \mathrm{~J})$ were killed with a peritoneal injection of a lethal dose of Euthesate ( $8 \mathrm{mg}$ sodium pentobarbital per mouse; Sanofi Santé Animale Benelux, Maassluis, The Netherlands). The animal committee of the VU University Amsterdam approved the use of mice in these experiments. Tibiae were collected and soft tissue removed. Cleaned tibiae were then ground in a mortar with $\alpha$-MEM (Gibco) supplemented with $10 \%$ FBS, $150 \mu \mathrm{g} / \mathrm{ml}$ penicillin, $125 \mu \mathrm{g} / \mathrm{ml}$ streptomycin, $1.25 \mu \mathrm{g} / \mathrm{ml}$ Fungizone, and heparin $(170 \mathrm{IU} / \mathrm{ml}$; Leo Pharmaceutical Products, Weesp, The Netherlands). The cell suspension was aspirated through a 21-gauge needle and filtered with a $100-\mu \mathrm{m}$-pore-size Cell Strainer filter (Falcon/Becton Dickinson, Franklin Lakes, NJ). Cells 
were then washed twice in culture medium and centrifuged for $10 \mathrm{~min}$ at $200 \times \mathrm{g}$, and $1 \times 10^{6}$ cells $/ \mathrm{cm}^{2}$ were seeded on top of mechanically loaded or static MLO-Y4 osteocytes.

Pulsatile Fluid Flow

MLO-Y4 osteocytes between passages 30 and 31 were collected with $0.25 \%$ trypsin (Difco Laboratories, Detroit, MI) and $0.1 \%$ EDTA (ethylenediaminetetraacetic acid; Sigma) in phosphate-buffered saline (PBS), and seeded at $1 \times 10^{3}$ cells $/ \mathrm{cm}^{2}$ on polylysine-coated $(50 \mu \mathrm{g} / \mathrm{ml}$; poly-Llysine hydrobromide; Sigma) glass slides $\left(15 \mathrm{~cm}^{2}\right)$ in $\alpha$-MEM with $5 \%$ FBS, $5 \%$ calf serum, and antibiotics. They were incubated overnight at $37^{\circ} \mathrm{C}$ with $5 \% \mathrm{CO}_{2}$ in air, then subjected to mechanical loading for $1 \mathrm{~h}$ by pulsating fluid flow (PFF) or kept under static control conditions. $\mathrm{PFF}$ at $5-\mathrm{Hz}$ pulse frequency was generated by pumping $13 \mathrm{ml}$ of culture medium in a pulsatile manner through a parallel-plate flow chamber $(65 \times 24 \times 0.3 \mathrm{~mm})$ containing the bone cells [16]. The mean applied fluid shear stress was $0.7 \mathrm{~Pa}$ with a pulse amplitude of $0.3 \mathrm{~Pa}$, and the estimated peak stress rate was $8.4 \mathrm{~Pa} / \mathrm{s}$ [17-19]. Control cultures were kept under stationary conditions in a petri dish containing $13 \mathrm{ml}$ flow medium under similar conditions as the experimental cultures, i.e., at $37^{\circ} \mathrm{C}$ in a humidified atmosphere of $5 \% \mathrm{CO}_{2}$ in air. After 1-h PFF or static control treatment, cells were postincubated without PFF for 1 or $6 \mathrm{~h}$ in $13 \mathrm{ml}$ of fresh medium. After 1-h PFF and at the end of each postincubation period, cells were lysed for total RNA isolation as described below.

\section{Osteoclast Formation}

MLO-Y4 osteocytes seeded at $1 \times 10^{3}$ cells $/ \mathrm{cm}^{2}$ on glass slides were either mechanically loaded by 1-h PFF $(0.7 \pm 0.3 \mathrm{~Pa}, 5 \mathrm{~Hz})$ or kept under static culture conditions. A total of $1 \times 10^{6}$ bone marrow cells $/ \mathrm{cm}^{2}$ were seeded on top of mechanically loaded or static MLO-Y4 osteocytes. They were kept in co-culture in $\alpha$-MEM (Gibco) supplemented with $10 \% \mathrm{FBS}, 150 \mu \mathrm{g} / \mathrm{ml}$ penicillin, $125 \mu \mathrm{g} / \mathrm{ml}$ streptomycin, and $1.25 \mu \mathrm{g} / \mathrm{ml}$ Fungizone. Culture medium was refreshed after 3 days. After 7 days of co-culture, cells were fixed in $4 \%$ formaldehyde in PBS for $10 \mathrm{~min}$. Fixed cells were washed with PBS and stained for tartrate-resistant acid phosphatase (TRACP) according to the manufacturer's instructions (Sigma). The number of TRACP-positive multinucleated (three or more nuclei per cell) and mononuclear cells were counted with a Leica DM IL microscope (Leica, Wetzlar, Germany) equipped with a $\times 20$ objective.

In a well-established monoculture osteoclastogenesis assay [3], bone marrow cells were seeded at $3 \times 10^{5}$ cells $/ \mathrm{cm}^{2}$ in 96-well plates in medium containing $30 \mathrm{ng} / \mathrm{ml}$ recombinant murine M-CSF (R\&D Systems, Minneapolis, MN), $20 \mathrm{ng} / \mathrm{ml}$ recombinant murine RANKL (RANKL-TEC; R\&D Systems), and 20, 24, 50, 60, or $75 \mathrm{ng} / \mathrm{ml}$ recombinant human OPG (Biovision, Mountain View, CA). Culture medium containing M-CSF, RANKL, and OPG was refreshed after 3 days. After 6 days of culture, cells were fixed, and TRACP-positive multinucleated cells were counted as described above.

\section{Effect of Recombinant MEPE on Osteoclast Formation}

MLO-Y4 osteocytes were seeded at $1 \times 10^{3}$ cells/well in 48-well tissue culture plate. After overnight attachment, osteocytes were treated for $24 \mathrm{~h}$ with $0.05,0.5$, or $5 \mu \mathrm{g} / \mathrm{ml}$ of recombinant human MEPE (R\&D Systems). A total of $1 \times 10^{6}$ bone marrow cells were seeded on top of recombinant human MEPE treated osteocytes. Co-culture was maintained in the presence of $0.05,0.5$, or $5 \mu \mathrm{g} / \mathrm{ml}$ recombinant human MEPE. After 7 days of co-culture, the number of TRACP-positive multinucleated and mononuclear cells was determined.

\section{Analysis of Gene Expression}

Real-time polymerase chain reaction was used to determine gene expression of MEPE, PHEX, RANKL, OPG, and the housekeeping gene GAPDH (all primers from Applied Biosystems, Foster City, CA). Total RNA was isolated with lysis buffer RA I (Macherey-Nagel, Düren, Germany) according to the manufacturer's instructions. cDNA synthesis was performed with $0.5-1 \mu \mathrm{g}$ of total RNA in a 20-ml reaction mixture consisting of 5 units of Transcriptor Reverse Transcriptase (Roche Diagnostics, Mannheim, Germany), 0.08 A260 units of random primers (Roche Diagnostics), $1 \mathrm{mmol} / \mathrm{l}$ of each dNTP (Invitrogen, Carlsbad, CA), and $1 \times$ concentrated Transcriptor RT reaction buffer (Roche Diagnostics). Real-time polymerase chain reaction reactions were performed with Taq-Man Gene Expression assays (TaqMan; Applied Biosystems) in an ABI Prism 7700 DNA sequence detector (Applied Biosystems). Gene expression values were normalized for housekeeping gene GAPDH.

\section{Statistical Analysis}

The Wilcoxon signed rank test was used for comparing the effect of mechanically loaded osteocytes and static osteocytes on the number of TRACP positive multinucleated cells. For statistical analysis of the data obtained from all other experiments, the paired Student's $t$-test was used. Differences were considered significant when $P<0.05$. 


\section{Results}

Osteocytes Support Osteoclast Formation from Bone Marrow Cells

Mouse bone marrow cells co-cultured on top of MLO-Y4 osteocytes for 7 days fused to form TRACP-positive multinucleated cells (TRACP ${ }^{+} \mathrm{MNCs}$ ) and mononuclear cells (Fig. 1a).

Mechanical Loading of Osteocytes Inhibits

Osteoclastogenesis from Bone Marrow Cells

Next, we investigated the effect of mechanical loading on osteoclast formation in a co-culture system of MLO-Y4 osteocytes and bone marrow cells. Osteocytes subjected to 1-h PFF stopped the formation of osteoclasts, as assessed by the number of TRACP ${ }^{+}$MNCs after 7 days of co-culture (Fig. 1b). One-hour PFF significantly inhibited the number of $\mathrm{TRACP}^{+} \mathrm{MNCs}$ (Fig. 1c) and mononuclear cells (Fig. 1d) compared to static conditions.

\section{PFF Affects MEPE but not PHEX Gene Expression}

To assess whether mechanical loading affects $M E P E$ and $P H E X$ gene expression, MLO-Y4 osteocytes were treated with or without 1-h PFF. Mechanical loading significantly upregulated $M E P E$ gene expression by 2.5 -fold after 1 -h PFF, but not at 1 or $6 \mathrm{~h}$ after termination of PFF (Fig. 2a). PFF did not affect $P H E X$ gene expression after $1-\mathrm{h}$ PFF treatment, nor at 1 or $6 \mathrm{~h}$ after termination of PFF (Fig. 2b). This suggests that $M E P E$ was relatively more upregulated than $P H E X$.

Role of OPG and RANKL in PFF-stimulated Osteoclastogenesis

To determine whether PFF affects osteoclastogenesisrelated gene expression, we analyzed gene expression of $R A N K L, O P G$, and $M-C S F$ in MLO-Y4 osteocytes after 1-h PFF. We found that PFF did not affect $M$-CSF gene expression after 1-h PFF (data not shown). PFF upregulated $R A N K L$ gene expression by 1.8 -fold after 1 -h PFF treatment (Fig. 3a). Similarly, PFF upregulated $O P G$ gene expression by 2.1-fold after 1-h PFF treatment (Fig. 3a). As a result, PFF decreased the RANKL/OPG ratio by $20 \%$ after 1-h PFF treatment (Fig. 3b). To determine whether a $20 \%$ change in the $R A N K L / O P G$ ratio affects osteoclastogenesis, we stimulated mouse bone marrow stromal cells with M-CSF and RANKL in the presence of different concentrations of OPG. We found that OPG dose dependently inhibited the number of TRACP ${ }^{+} \mathrm{MNCs}$ (Fig. 3c).
Fig. 1 Mechanical loading of osteocytes inhibits osteoclastogenesis from bone marrow cells. a TRACPpositive multinucleated cells (arrows) and TRACP-positive mononuclear cells (arrowheads) after 7 days of co-culture of static MLO-Y4 osteocytes and mouse bone marrow cells. b MLO-Y4 osteocytes subjected to 1-h PFF nihilized the formation of TRACP-positive multinucleated cells after 7 days of co-culture with bone marrow cells. Original magnification, $\times 20$. The number of $\mathbf{c}$ TRACPpositive multinucleated and d TRACP-positive mononuclear cells was significantly inhibited due to 1-h PFF exposure of MLO-Y4 osteocytes. Values are means \pm SEM from 3 separate experiments. $P F F$ pulsating fluid flow, Static static control cultures. * Significant effect of PFF, $P<0.05$
A

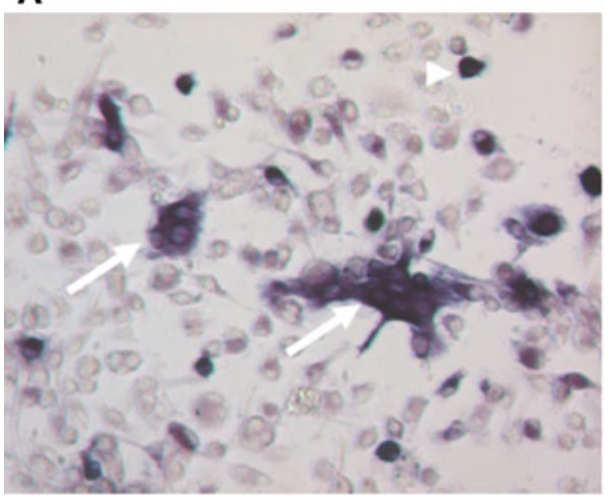

Static

C

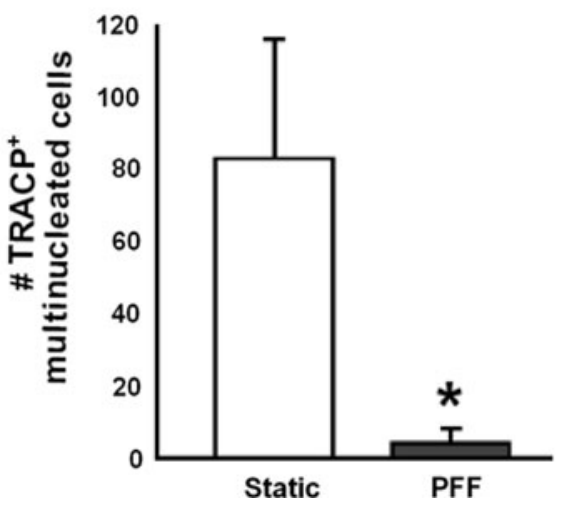

B
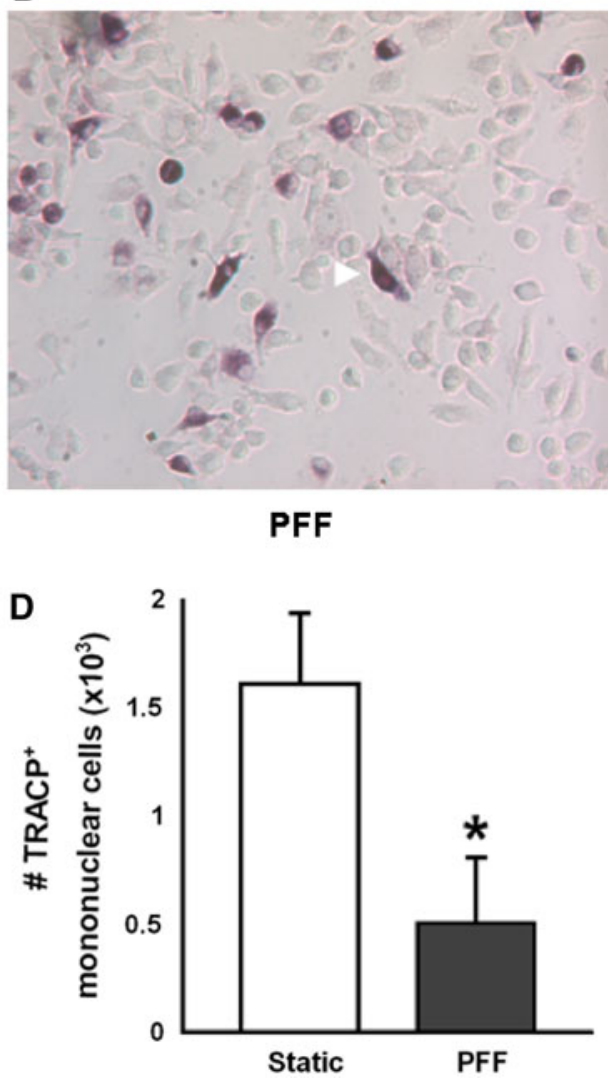


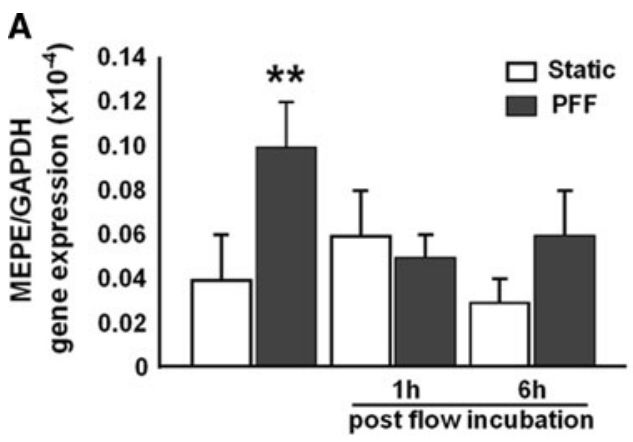

Fig. 2 PFF treatment upregulates MEPE but not PHEX gene expression by MLO-Y4 osteocytes. a PFF significantly upregulated $M E P E$ gene expression after 1-h PFF, but not at 1 or $6 \mathrm{~h}$ after termination of PFF. b PFF did not affect PHEX gene expression after 1-h PFF, or 1 and $6 \mathrm{~h}$ after termination of PFF. Data are expressed as
B

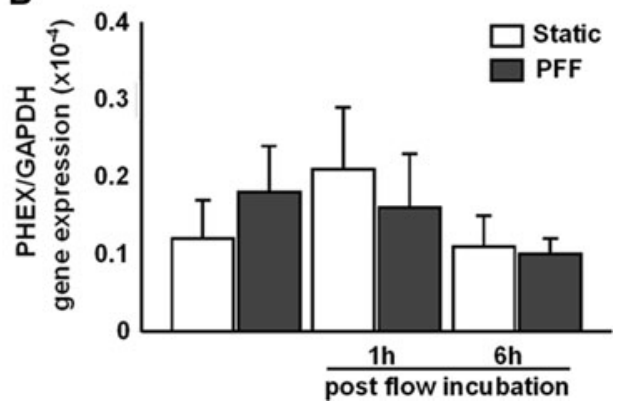

relative gene expression normalized for $G A P D H$ gene expression. Values are means \pm SEM from 4 separate experiments. $P F F$ pulsating fluid flow, Static static control cultures. ** Significant effect of PFF, $P<0.01$
Fig. 3 Role of OPG and RANKL in PFF-stimulated osteoclastogenesis. a PFF significantly upregulated $R A N K L$ and $O P G$ gene expression after 1-h PFF treatment. b PFF downregulated the $R A N K L / O P G$ ratio after 1-h PFF treatment. $\mathbf{c}$ Effect of OPG on M-CSF and RANKL-induced osteoclastogenesis. OPG dosedependently inhibited the number of TRACP-positive multinucleated cells. A $20 \%$ decrease in the RANKL/OPG ratio (OPG increased from $20 \mathrm{ng} / \mathrm{ml}$ to $24 \mathrm{ng} / \mathrm{ml}$ ) was already sufficient to inhibit the number of TRACP-positive multinucleated cells. Values are means \pm SEM from 3 or more separate experiments. PFF pulsating fluid flow, Static static control cultures. $*$ Significant effect of PFF, $P<0.05$
A
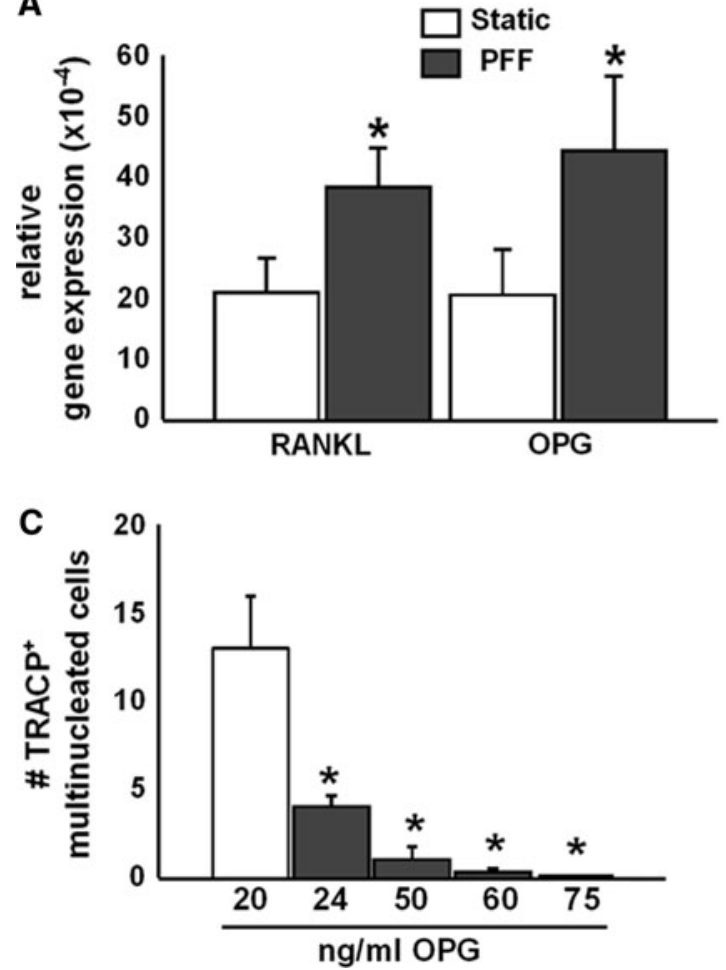

B

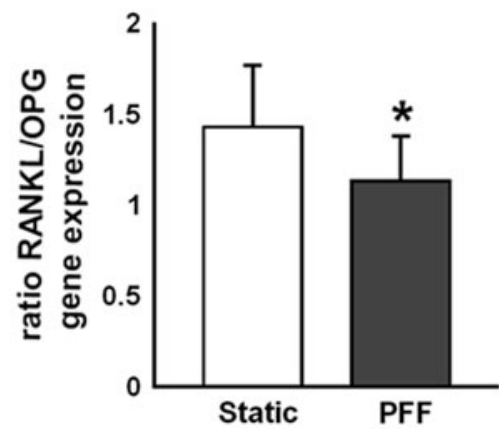

Importantly, we found that a decrease in the RANKL/OPG ratio of $20 \%$ was already sufficient to inhibit the number of $\mathrm{TRACP}^{+} \mathrm{MNCs}$ in our osteoclastogenesis assay (Fig. 3c).

\section{Recombinant Human MEPE Inhibits Osteocyte-} stimulated Osteoclastogenesis

Mechanical loading of osteocytes with 1-h PFF significantly increased MEPE gene expression. We also studied whether recombinant human MEPE could modulate osteocyte-stimulated osteoclastogenesis. We found that MEPE at $5 \mu \mathrm{g} / \mathrm{ml}$ inhibited the number of TRACP-positive multinucleated cells (Fig. 4a) and TRACP-positive mononuclear cells (Fig. 4b).

\section{Discussion}

Osteocytes are the predominant bone cells and the professional mechanosensors of bone [2]. In response to mechanical loading, osteocytes communicate with each other and with other types of cells, including osteoblasts, bone-lining cells, and osteoclasts, through the canalicular network in which nutrients and other soluble factors are 


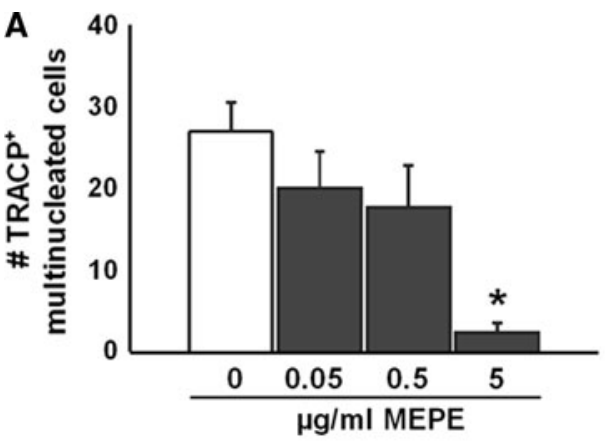

Fig. 4 Effect of recombinant human MEPE on osteocyte-stimulated osteoclastogenesis. Recombinant MEPE at $5 \mu \mathrm{g} / \mathrm{mL}$ dowregulated the number of a TRACP-positive multinucleated cells, and b TRACP-

transported by fluid flow. Osteocytes are therefore considered to be the orchestrators of bone adaptation to mechanical loading [1-4].

Bone resorption is suppressed in response to mechanical loading of bone in vivo [20, 21]. We have shown earlier that conditioned medium from osteocytes subjected to PFF inhibits osteoclast formation and function [3]. It was, however, not demonstrated which molecules in the conditioned medium were responsible for the inhibitory effect on osteoclast formation and function. One of the molecules in conditioned medium responsible for the inhibitory effect could be MEPE. MEPE $^{-/-}$mice have a marked age dependent increased bone mass and increased in vivo mineral apposition rate without any effect on osteoclast number [22]. In contrast, overexpression of MEPE decreased osteoclast number and activity in a murine model [23]. The purpose of the current study was to investigate whether mechanical loading of osteocytes affects osteocyte-stimulated osteoclastogenesis by involvement of MEPE.

Osteocytes extend cell processes into bone marrow space [24]. It is likely that osteocytes communicate directly with the osteoclast precursors in the bone marrow space via dendritic processes. Humoral factors produced by osteocytes are released through the canaliculi into the bone marrow space, where they may regulate the differentiation and activity of osteoclasts. Therefore, we used a co-culture model of MLO-Y4 osteocytes and mouse bone marrow cells that likely mimics the in vivo situation. Recently, Tatsumi et al. [6] showed that osteocyte-ablated mice exhibit almost complete resistance to osteoclastic bone resorption in response to unloading. These results indicate that osteocytes play a crucial role in sensing the local changes in mechanical strains evoked by unloading and/or play a role in transmitting pro-osteoclastogenic signals to stimulate bone resorption in response to mechanical unloading [6]. On the other hand, osteocytes are known to produce several factors in response to mechanical loading

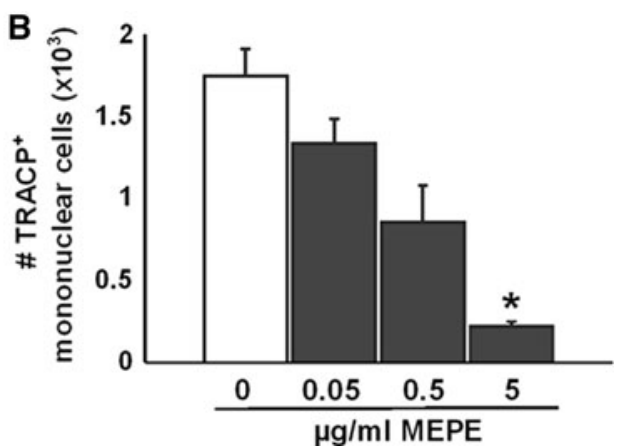

positive mononuclear cells after 7 days of co-culture of MLO-Y4 osteocytes and mouse bone marrow cells. Values are means \pm SEM from 3 separate experiments. * Significant effect of PFF, $P<0.05$

that can inhibit osteoclastic bone resorption [3, 25]. Mechanical loading of osteocytes upregulates transforming growth factor- $\beta$, which inhibits osteoclastic bone resorption as well [25]. Vascular endothelial growth factor, which increases osteoclastogenesis in vitro [26], has also been found to be increased after mechanical loading of primary bone cells [27]. Osteocytes produce high levels of nitric oxide in response to loading in the form of a physiologic fluid shear stress [28, 29], and nitric oxide inhibits osteoclast activity $[3,30]$. Thus, osteocytes have different ways to modulate osteoclastogenesis and osteoclast activity, and MEPE could be one of those several factors produced in response to mechanical loading by osteocytes modulating osteoclastogenesis and osteoclast activity.

Osteocytes transduce opposite signals depending on the nature and direction of mechanical input, i.e., an antiosteoclastogenic signal (under normal loading) versus a pro-osteoclastogenic signal (in response to unloading) [6]. In our study, we found that osteocytes support osteoclast formation under static culture conditions, whereas mechanical loading of osteocytes by PFF significantly inhibited the formation of osteoclasts from bone marrow cells. This is consistent with previous findings that mechanical loading of MLO-Y4 osteocytes inhibits osteoclast formation from RAW264.7 mouse monocytes/macrophages [5]. Mechanical loading of ST-2 murine stromal bone marrow cells decreases osteoclastogenesis from RAW264.7 cells through a decrease in the RANKL/OPG ratio [31]. Osteoclasts in vivo are likely derived from osteoclast precursors present in bone marrow spaces [32]. In our study, we used mouse bone marrow cells as a source of osteoclast precursors. Here we show for first time that mechanical loading of osteocytes inhibits osteoclast formation from bone marrow cells, which likely also occurs in vivo.

Binding of PHEX to MEPE prevents cathepsin-mediated cleavage of MEPE and the concomitant release of ASARM peptide from MEPE [14]. We found that 
mechanical loading of osteocytes by PFF upregulated $M E P E$ but not PHEX gene expression. $O P G$ gene expression has been shown to be significantly increased in bone marrow stromal cells incubated with ASARM-PO4 [14]. $M E P E$ gene expression was relatively more upregulated than PHEX gene expression. Assuming the changes in gene expression reflect changes in protein expression, this would make MEPE available for cathepsin cleavage to produce ASARM peptides and thereby upregulate $O P G$. We suggest that the loading-induced shift in the balance between $M E P E$ and PHEX expression by osteocytes was responsible for the loading-induced increase in $O P G$ gene expression via the production of ASARM peptides. This could explain our finding that mechanical loading of osteocytes downregulated the RANKL/OPG ratio immediately after PFF treatment. Because the changes in the RANKL/OPG ratio after $\mathrm{PFF}$ were relatively small, we simulated the PFF-induced changes in the RANKL/OPG ratio and studied the effect on osteoclastogenesis. We found that a decrease in the RANKL/OPG ratio by $20 \%$ strongly inhibited osteoclastogenesis, which suggests that the PFFinduced inhibition of osteoclastogenesis can be attributed to a loading-induced decrease in RANKL/OPG ratio.

Because we found that mechanical loading of osteocytes by PFF upregulated MEPE but not PHEX gene expression, we studied whether recombinant human MEPE modulated osteocyte-stimulated osteoclastogenesis. Importantly, the amount of MEPE protein present in conditioned medium after PFF treatment of osteocytes might not reflect the amount of MEPE protein seen by osteoclast precursors in our co-culture model. We have not used conditioned medium of osteocytes to inhibit osteoclastogenesis, but osteoclast precursors were seeded on top of osteocytes. The osteoclast precursors that were thus in direct contact with the osteocytes were likely exposed to high local MEPE protein concentrations. Serum MEPE levels in healthy adult humans vary between 0.02 and $1.3 \mu \mathrm{g} / \mathrm{ml}$ [13]. Because MEPE is predominantly expressed by osteocytes in human bone, it is likely that local MEPE levels in bone are even higher than the serum MEPE levels in healthy adult humans. We found that recombinant human MEPE inhibited osteocyte-stimulated osteoclastogenesis, which is in accordance with findings by others that recombinant human MEPE decreases parathyroid hormone-related protein and 1,25-dihydroxy vitamin $\mathrm{D}_{3}$-induced osteoclast formation from mouse bone marrow cells [33]. Although we cannot prove that MLO-Y4 cells produced MEPE at a concentration of $0.05-5 \mu \mathrm{g} / \mathrm{ml}$, it can be appreciated that PFF upregulates MEPE gene expression in osteocytes, and that recombinant MEPE can inhibit osteoclastogenesis at concentrations lower than the MEPE concentrations likely present in bone. Our results indicate that
MEPE may contribute to the mechanical loading-induced inhibition of osteoclastogenesis.

In summary, osteocytes subjected to 1-h PFF significantly inhibited the formation of osteoclasts from bone marrow cells. On the basis of our results, we speculate that MEPE might be a soluble factor produced after mechanical loading of osteocytes, leading to the inhibition of osteoclastogenesis. This supports the regulatory role for osteocytes in osteoclast formation and bone resorption. Increased understanding of communication between mechanically loaded osteocytes and osteoclasts will help to clarify bone remodeling in response to mechanical loading, and it might contribute to new treatment modalities for diseases where this communication might be disturbed, such as osteoporosis or osteopetrosis. Thus, MEPE could be a potential therapeutic agent for treatment of diseases such as osteoporosis.

Acknowledgments The work of R.N.K. was supported by a grant from the University of Amsterdam. The Research Institute MOVE of the VU University Amsterdam supported the work of A.D.B. We thank T. J. de Vries and R. G. Bacabac for helpful discussions.

\section{Disclosures None.}

Open Access This article is distributed under the terms of the Creative Commons Attribution Noncommercial License which permits any noncommercial use, distribution, and reproduction in any medium, provided the original author(s) and source are credited.

\section{References}

1. Burger EH, Klein-Nulend J, Van der Plas A, Nijweide PJ (1995) Function of osteocytes in bone-their role in mechanotransduction. J Nutr 125:2020S-2023S

2. Burger EH, Klein-Nulend J (1999) Mechanotransduction in bone-role of the lacunocanalicular network. FASEB J 13:S101S112

3. Tan SD, de Vries TJ, Kuijpers-Jagtman AM, Semeins CM, Everts V, Klein-Nulend J (2007) Osteocytes subjected to fluid flow inhibit osteoclast formation and bone resorption. Bone 41:745-751

4. Vezeridis PS, Semeins CM, Chen Q, Klein-Nulend J (2006) Osteocytes subjected to pulsating fluid flow regulate osteoblast proliferation and differentiation. Biochem Biophys Res Commun 348:1082-1088

5. You L, Temiyasathit S, Lee P, Kim CH, Tummala P, Yao W, Kingery W, Malone AM, Kwon RY, Jacobs CR (2008) Osteocytes as mechanosensors in the inhibition of bone resorption due to mechanical loading. Bone 42:172-179

6. Tatsumi S, Ishii K, Amizuka N, Li M, Kobayashi T, Kohno K, Ito M, Takeshita S, Ikeda K (2007) Targeted ablation of osteocytes induces osteoporosis with defective mechanotransduction. Cell Metab 5:464-475

7. Lerner U (2004) New molecules in the tumor necrosis factor ligand and receptor superfamilies with importance for physiological and pathological bone resorption. Crit Rev Oral Biol Med 15:64-81 
8. Teitelbaum S (2007) Osteoclasts: what do they do and how do they do it? Am J Pathol 170:427-435

9. Nagai M, Sato N (1999) Reciprocal gene expression of osteoclastogenesis inhibitory factor and osteoclast differentiation factor regulates osteoclast formation. Biochem Biophys Res Commun 257:719-723

10. Nampei A, Hashimoto J, Hayashida K, Tsuboi H, Shi K, Tsuji I, Miyashita H, Yamada T, Matsukawa N, Matsumoto M, Morimoto S, Ogihara T, Ochi T, Yoshikawa H (2004) Matrix extracellular phosphoglycoprotein (MEPE) is highly expressed in osteocytes in human bone. J Bone Miner Metab 22:176-184

11. Gluhak-Heinrich J, Pavlin D, Yang W, MacDougall M, Harris S (2007) MEPE expression in osteocytes during orthodontic tooth movement. Arch Oral Biol 52:684-690

12. Hayashibara T, Hiraga T, Yi B, Nomizu M, Kumagai Y, Nishimura R, Yoneda T (2004) A synthetic peptide fragment of human MEPE stimulates new bone formation in vitro and in vivo. J Bone Miner Res 19:455-462

13. Jain A, Fedarko NS, Collins MT, Gelman R, Ankrom MA, Tayback M, Fisher LW (2004) Serum levels of matrix extracellular phosphoglycoprotein (MEPE) in normal humans correlate with serum phosphorus, parathyroid hormone and bone mineral density. J Clin Endocrinol Metab 89:4158-4161

14. Martin A, David V, Laurence JS, Schwarz PM, Lafer EM, Hedge AM, Rowe PS (2008) Degradation of MEPE, DMP1, and release of SIBLING ASARM-peptides (minhibins): ASARM-peptide(s) are directly responsible for defective mineralization in HYP. Endocrinology 149:1757-1772

15. Kato Y, Windle JJ, Koop BA, Mundy GR, Bonewald LF (1997) Establishment of an osteocyte-like cell line, MLO-Y4. J Bone Miner Res 12:2014-2023

16. Bakker AD, Soejima K, Klein-Nulend J, Burger EH (2001) The production of nitric oxide and prostaglandin $\mathrm{E}(2)$ by primary bone cells is shear stress dependent. J Biomech 34:671-677

17. Bacabac RG, Smit TH, Mullender MG, Dijcks SJ, Van Loon JJWA, Klein-Nulend J (2004) Nitric oxide production by bone cells is fluid shear stress rate dependent. Biochem Biophys Res Commun 315:823-829

18. Bacabac RG, Smit TH, Cowin SC, Van Loon JJWA, Nieuwstadt FT, Heethaar R, Klein-Nulend J (2005) Dynamic shear stress in parallel-plate flow chambers. J Biomech 38:159-167

19. Klein-Nulend J, van der Plas A, Semeins CM, Ajubi NE, Frangos JA, Nijweide PJ, Burger EH (1995) Sensitivity of osteocytes to biomechanical stress in vitro. FASEB J 9:441-445

20. Duncan RL, Turner CH (1995) Mechanotransduction and the functional response of bone to mechanical strain. Calcif Tissue Int 57:344-358

21. Hillam RA, Skerry TM (1995) Inhibition of bone resorption and stimulation of formation by mechanical loading of the modeling rat ulna in vivo. J Bone Miner Res 10:683-690
22. Gowen LC, Petersen DN, Mansolf AL, Qi H, Stock JL, Tkalcevic GT, Simmons HA, Crawford DT, Chidsey-Frink KL, Ke HZ, McNeish JD, Brown TA (2003) Targeted disruption of the osteoblast/osteocyte factor 45 gene (OF45) results in increased bone formation and bone mass. J Biol Chem 278:1998-2007

23. David V, Martin A, Hedge AM, Rowe PS (2009) MEPE is a new bone renal hormone and vascularization modulator. Endocrinology 150:4012-4023

24. Kamioka H, Honjo T, Takano-Yamamoto T (2001) A threedimensional distribution of osteocyte processes revealed by the combination of confocal laser scanning microscopy and differential interference contrast microscopy. Bone 28:145-149

25. Heino TJ, Hentunen TA, Väänänen HK (2002) Osteocytes inhibit osteoclastic bone resorption through transforming growth factorbeta: enhancement by estrogen. J Cell Biochem 85:185-197

26. Sipola A, Nelo K, Hautala T, Ilvesaro J, Tuukkanen J (2006) Endostatin inhibits VEGF-A induced osteoclastic bone resorption in vitro. BMC Musculoskelet Disord 7:56

27. Faure C, Linossier MT, Malaval L, Lafage-Proust MH, Peyroche S, Vico L, Guignandon A (2008) Mechanical signals modulated vascular endothelial growth factor-A (VEGF-A) alternative splicing in osteoblastic cells through actin polymerisation. Bone 42:1092-1101

28. Klein-Nulend J, Semeins CM, Ajubi NE, Nijweide PJ, Burger EH (1995) Pulsating fluid flow increases nitric oxide (NO) synthesis by osteocytes but not periosteal fibroblasts-correlation with prostaglandin upregulation. Biochem Biophys Res Commun 217:640-648

29. Zaman G, Pitsillides AA, Rawlinson SC, Suswillo RF, Mosley JR, Cheng MZ, Platts LA, Hukkanen M, Polak JM, Lanyon LE (1999) Mechanical strain stimulates nitric oxide production by rapid activation of endothelial nitric oxide synthase in osteocytes. J Bone Miner Res 14:1123-1131

30. Palmer RM, Ferrige AG, Moncada S (1987) Nitric oxide release accounts for the biological activity of endothelium-derived relaxing factor. Nature 327:524-526

31. Kim CH, You L, Yellowley CE, Jacobs CR (2006) Oscillatory fluid flow-induced shear stress decreases osteoclastogenesis through RANKL and OPG signaling. Bone 39:1043-1047

32. De Vries TJ, Schoenmaker T, Hooibrink B, Leenen PJ, Everts V (2009) Myeloid blasts are the mouse bone marrow cells prone to differentiate into osteoclasts. J Leukoc Biol 85:919-927

33. Hayashibara T, Hiraga T, Sugita A, Wang L, Hata K, Ooshima T, Yoneda T (2007) Regulation of osteoclast differentiation and function by phosphate: potential role of osteoclasts in the skeletal abnormalities in hypophosphatemic conditions. J Bone Miner Res 22:1743-1751 